\title{
Genomic characterization of high- recurrence risk papillary thyroid carcinoma in a southern Chinese population
}

Min Li ${ }^{1}$, Haitao Jia ${ }^{2}$, Qiuqin Qian', Peng Wen ${ }^{1}$, Chuan Chen², Yaqiong Hua ${ }^{2}$, Kai Wang ${ }^{2}$, Wenyong Zhang ${ }^{3}$ and Feng Shi $^{*}{ }^{*}$ D

\begin{abstract}
Background: The objective of this study was to investigate genetic variations and the relationships between these genetic variations and clinicopathological features of high-recurrence risk papillary thyroid carcinoma in a southern Chinese population.

Methods: One hundred sixty-eight patients of high-recurrence risk papillary thyroid carcinoma were recruited for this study from 2017 to 2018. Formalin-fixed paraffin-embedded tissue and the data of clinicopathological characteristics were all collected and analyzed from these patients. We used next-generation sequencing technology to investigate the targeted gene mutations and gene fusions of the pathology specimens.

Results: The frequency of candidate tumor driver gene mutation was $85.1 \%$ in 143 patients, including BRAF V600E mutation in 119 patients(70.8\%), RET fusion in 13 patients(7.7\%), TERT promoter mutations in 11 patients(6.5\%), RAS (HRAS, NRAS, KRAS) gene mutations in 10 patients(6.0\%), and other mutations involving TP53, PIK3CA, AKT1, PTEN and NTRK1. Concomitant presence of more than two genetic aberrations was seen in 27 patients (16.1\%). Our study showed that BRAF V600E mutation is highly correlated with conventional PTC $(p<0.001)$, BRAF V600E and TERT promoter mutation duet was associated with older patient age $(>45, p=0.003)$ and higher disease stage of III or IV ( $p=0.002)$. RAS gene and BRAF V600E co-mutations were only seen in multifocal PTC $(p=0.015)$.

Conclusion: In our high-recurrence risk PTC cohort, most patients had more than one driver gene aberration. Coexistence of BRAF V600E with TERT promoter mutations or with RAS mutations were significantly correlated with worse clinicopathological characteristics.
\end{abstract}

Keywords: Papillary thyroid carcinoma, BRAF, TERT, RET fusion, RAS, TP53, Driver gene

\footnotetext{
* Correspondence: smsm3028@sina.com

'Department of Nuclear Medicine, The Affiliated Cancer Hospital of Xiangya

School of Medicine, Central South University, and Hunan Cancer Hospital,

Changsha 410013, China

Full list of author information is available at the end of the article
}

(C) The Author(s). 2020 Open Access This article is licensed under a Creative Commons Attribution 4.0 International License, which permits use, sharing, adaptation, distribution and reproduction in any medium or format, as long as you give appropriate credit to the original author(s) and the source, provide a link to the Creative Commons licence, and indicate if changes were made. The images or other third party material in this article are included in the article's Creative Commons licence, unless indicated otherwise in a credit line to the material. If material is not included in the article's Creative Commons licence and your intended use is not permitted by statutory regulation or exceeds the permitted use, you will need to obtain permission directly from the copyright holder. To view a copy of this licence, visit http://creativecommons.org/licenses/by/4.0/ The Creative Commons Public Domain Dedication waiver (http://creativecommons.org/publicdomain/zero/1.0/) applies to the data made available in this article, unless otherwise stated in a credit line to the data. 


\section{Introduction}

The incidence of thyroid cancer has increased throughout the world in the last few decades [1]. In the United States, its incidence has seen a 3.8-fold increase since 1973 [2], and in China, a study reported that more than a 3-fold increase in thyroid cancer incidence from 1983 to 2007 in Shanghai [3]. Papillary thyroid cancer (PTC) is the most common type of thyroid cancer and makes up about $85-90 \%$ of all thyroid cancer cases. Generally, PTC patients have a favorable prognosis with average 10 -year survival of over $90 \%$. However, recurrence remains relatively common, particularly for invasive PTC and cancer with BRAF V600E mutation [4].

A recent study of the molecular pathogenesis of PTC has revealed several genetic mutations that can be used as diagnostic markers as well as therapeutic targets [5]. The most common mutations in PTC, including BRAF point mutations, $R A S$ point mutations, and RET gene rearrangements, perturb cell signaling in the mitogenassociated protein kinase (MAPK) pathway, leading to inappropriate cell growth and survival [6]. BRAF V600E mutation is the most common mutation seen in PTC, affecting approximately 50-60\% of all PTC cases [7]. $B R A F$ V600E mutation has been associated with more aggressive tumor characteristics, such as capsular invasion, lymph node metastasis, distal metastasis and recurrence [8]. TERT promoter mutations, most commonly C228T and C250T, have been associated with poor patient outcomes [9]. Although less frequent, mutations in PI3K/AKT pathway genes such as PIK3CA, and tumor suppressor genes such as TP53 and PTEN have been identified in PTC, indicating complex genetic aberrations disturbing cellular growth and survival signals and contributing to the pathogenesis of PTC [10].

As thyroid cancer incidence has increased rapidly in China in recent years, and targeted therapies have become available in China to treat various types of cancer [11], we set out to characterize the genetic mutations of PTC in a high-recurrent risk cohort from Southern China to better understand the genetic-clinicopathologic correlation of this disease andprovide insight into the target therapy options.

\section{Material and Method \\ Thyroid samples}

One hundred sixty-eight patients of high-recurrence risk papillary thyroid carcinoma were recruited for this study from 2017 to 2018, They all had received radioiodine therapy. These patients were designated as the highrecurrence risk PTC group by clinical diagnosis of lymph node metastasis, capsular invasion or extrathyroidal invasion. Formalin-fixed paraffin-embedded (FFPE) tissue were collected from surgery at the Department of Pathology, Hunan Cancer Hospital. The ethics committee of
Hunan Cancer Hospital passed ethical approval of this study, and the informed consents were confirmed by all participants before submitting this manuscript.

\section{DNA isolation}

Genomic DNA were extracted from $15 \times 5 \mu \mathrm{m}$ thick tissue sections of FFPE tumor tissue using QIAamp DNA FFPE Tissue Kit according to the manufacturer's instructions (Qiagen, Hilden, Germany). The percentage of tumor cells in the hematoxylin and eosin-stained slides were $>20 \%$ of the total tissue area, to ensure sufficient tumor DNA required for next generation sequencing. DNA concentrations were measured by a NanoDrop 2000 Spectrophotometer (Thermo Fisher Scientific, Waltham, MA, USA). All DNA concentrations were greater than $30 \mathrm{ng} / \mathrm{L}$, and $100 \mathrm{ng}$ DNA were used for NGS library construction.

\section{NGS library preparation}

For NGS library preparation, DNA was fragmented using Covaris M220. Fragments of 200-400 bp in size were selected by beads (Agencourt AMPure XP kit; Beckman Coulter, Inc., Brea, CA, USA), then followed by end repair, phosphorylation and adaptor ligation. Then the library was pre-amplified with a high fidelity enzyme, followed by hybridization with a capture probe panel consisting of 14 PTC related genes (Supplementary 1), including $10 \mathrm{mu}$ tated genes (BRAF, TERT, NRAS, HRAS, KRAS, PIK3CA, PTEN, AKT1, TP53, CTNNB1) and 4 fusion genes (RET, $A L K, P A X 8, N T R K 1)$, hybrid selection with magnetic beads and PCR amplification.

\section{Targeted DNA sequencing}

After QC and quantification by Agilent 2100 Bioanalyzer (Agilent Technologies) and Qubit ${ }^{\dagger} 3.0$ Fluorometer (Invitrogen), the capture-based targeted library were deep sequenced on NextSeq 500 (Illumina) with pair-end reads $(2 \times 150$ cycles $)$. The raw sequence data were mapped to the human genome (hg19) using BWA Aligner 0.7.10.

\section{Results}

Tumor tissues from 168 cases of thyroid papillary carcinoma were analyzed by next-generation sequencing. The patients live in Southern China and of Han ethnicity. Thirty-seven were males and 131 were females. The average age of cancer onset was 38.8 years for males and 39.8 years for females. The general characteristics of the study population are summarized in Table 1 . Most of the PTC patients were diagnosed with conventional PTC (92.9\%, 156/168), the remaining patients were diagnosed with follicular variant PTC $(7.1 \%, 12 / 168)$. The clinicopathological information of 168 patients were collected and shown in Supplementary Table 2. 
Table 1 Clinical characteristics of 168 PTC patients in southern Chinese populations

\begin{tabular}{lc}
\hline Characteristics & $N=168$ \\
\hline Gender & No. $(\%)$ \\
Female & $131(78.0)$ \\
Male & $37(22.0)$ \\
Age & \\
$<45$ & $107(63.7)$ \\
$\geq 45$ & $61(36.3)$ \\
Subtypes & \\
conventional PTC & $156(92.9)$ \\
follicular variant PTC & $12(7.1)$ \\
Lymph node metastasis & $155(92.3)$ \\
Yes & $13(7.7)$ \\
No & \\
AJCC disease stage & $109(64.9)$ \\
I + II & $59(35.1)$ \\
III + IV & \\
Lesion number & $78(46.4)$ \\
Single lesion & $90(53.6)$ \\
Multiple lesions &
\end{tabular}

The frequency of candidate tumor driver gene mutation was $85.1 \%(143 / 168)$. The results showed that BRAF V600E was the most common mutation type in PTC with a mutation frequency of $70.8 \%$ (119/168). The next most frequent mutations in this patient population was RET fusion, which was seen in $7.7 \%(13 / 168)$ of patients. TERT promoter mutations $\mathrm{C} 228 \mathrm{~T}$ or $\mathrm{C} 250 \mathrm{~T}$ were found in $6.5 \%(11 / 168)$ of patients. RAS (HRAS, NRAS, KRAS) gene mutations had a frequency of $6.0 \%(10 / 168)$. Other mutations included TP53, PIK3CA, AKT1, PTEN and NTRK1 fusion (Table 2).

In this study, fusion gene mutations were detected in 15 PTC cases $(8.9 \%, 15 / 168)$, of which 13 involved RET fusions, and the remaining 2 cases involved NTRK1 fusions. NCOA4-RET fusion was seen in 7 cases and CCDC6-RET was seen in 5 cases. ERC1-RET was seen in one case. NTRK1 fusion mutations were seen in 2 cases. No $A L K$ or PAX8-PPARY fusions were detected. (Fig. 1).

Mutations in the TERT promoter region were the third most common mutation type in this study, primarily TERT C228T and C250T mutations. There were 10 cases with $\mathrm{C} 228 \mathrm{~T}$ mutation and 1 case with C250T mutation. The next most frequently mutated gene in our study was the RAS family genes, with 10 cases in total: NRAS (4 cases), HRAS (4 cases), KRAS (2 cases). Mutations in TP53 (5 cases), PIK3CA (3 cases), AKT1 (3 cases), $P T E N(2$ cases $)$ were also detected in this study.
Table 2 Genetic variants of 168 PTC patients in southern Chinese populations

\begin{tabular}{lll}
\hline Genetic Variants & This study $(N=168)$ & $\%$ \\
\cline { 2 - 3 } & No. & 70.8 \\
\hline BRAF V600E & 119 & 8.9 \\
Gene fusion status & 15 & 7.7 \\
RET fusion & 13 & 1.2 \\
NTRK1 fusion & 2 & 6.5 \\
TERT status & 11 & 6.0 \\
C228T & 10 & 0.5 \\
C250T & 1 & 6.0 \\
RAS status & 10 & 2.4 \\
NRAS & 4 & 2.4 \\
HRAS & 4 & 1.2 \\
KRAS & 2 & 3.0 \\
TP53 mutation & 5 & 2.4 \\
PIK3CA mutation & 4 & 1.8 \\
AKT1 mutation & 3 & 1.2 \\
PTEN mutation & 2 &
\end{tabular}

Twenty-seven cases $(16.1 \%, 27 / 168)$ of co-mutation with $B R A F$ V600E were identified in this study, including one patient with $B R A F+P I K 3 C A+K R A S$ triple mutations. Types of mutation Included: BRAF V600E + TERT (10 cases), BRAF V600E + RAS (7 cases), BRAF V600E + TP53 (3 cases), BRAF V600E + PIK3CA (3 cases), BRAF $\mathrm{V} 600 \mathrm{E}+A K T 1$ (2 cases), BRAF V600E + PTEN (1 case), respectively (Table 3 ).

\section{Discussion}

Although PTC typically has a fairly good prognosis, approximately $30 \%$ of patients will experience disease progression or recurrence [12]. Studies have identified several genes, e.g. BRAF, TERT, RAS, RET, that play important roles in disease initiation or progression [10]. In our study, we chose to characterize the mutations in a cohort of high-recurrence risk PTC patients and examined the correlation between genetic mutations and clinicopathologic features. We found that BRAF V600E alone and co-mutations status of BRAF + TERT, BRAF+ $R A S$ showed correlation with age, disease stage and lesion number (Table 4).

$B R A F$ V600E is a driver mutation that plays an important role in PTC diagnosis, prognosis and treatment method selection. Currently, many studies have shown that BRAF V600E mutation correlates with other factors of poor prognosis, including patient age, bigger tumor size, extracapsular invasion, multifocality, lymph node metastasis, distant metastasis and higher TNM stage [13-15]. Our study showed that BRAF V600E mutation is highly correlated with PTC tumor type $(p<0.001)$. 


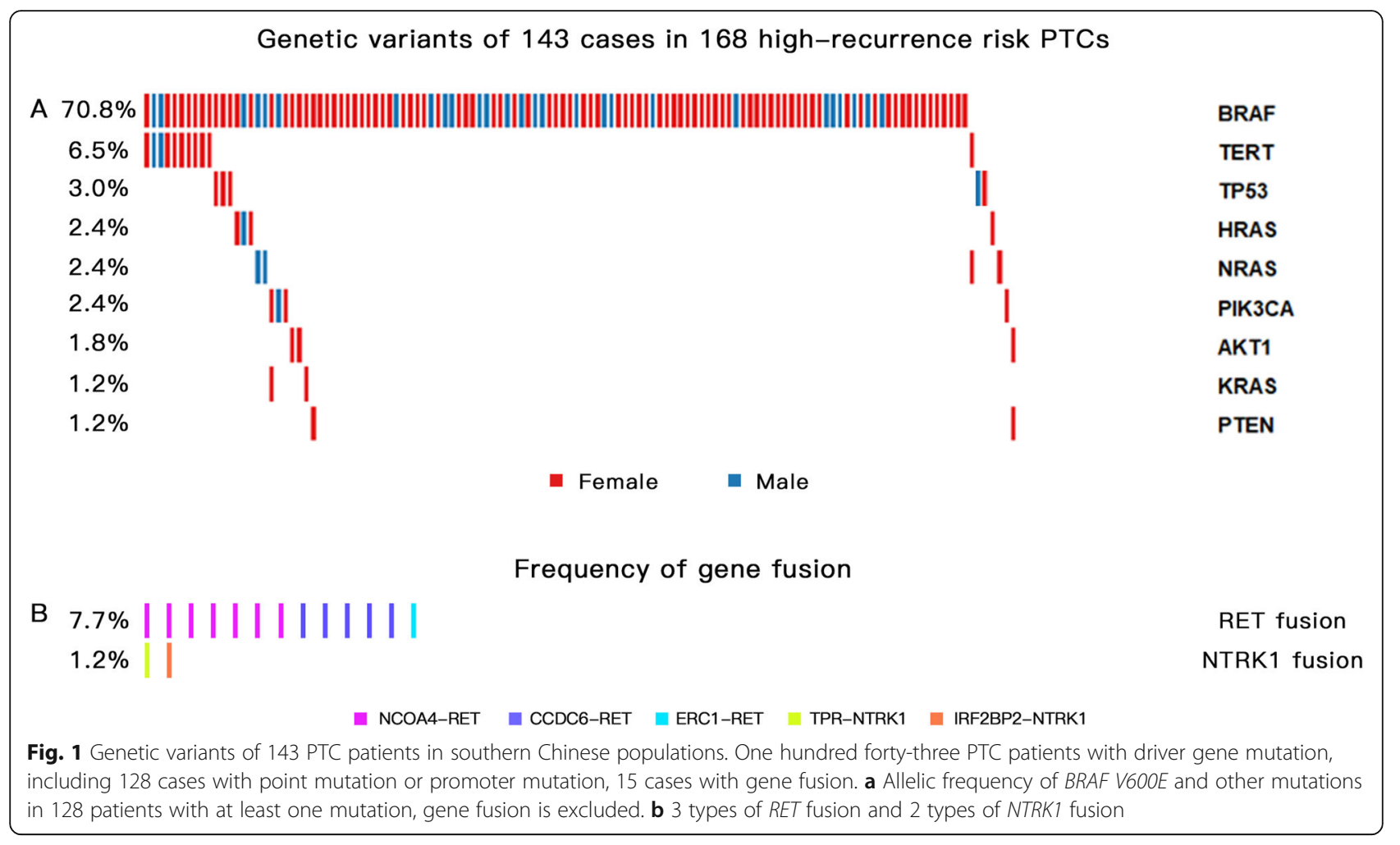

However, it is not correlated with gender, age, lymph node involvement, AJCC disease stage (AJCC 7th Edition), or lesion numbers. Zhang et al. also reported in a study of Chinese PTC patients that $88.3 \%$ of conventional PTC patients had BRAF V600E mutation [16]. Liang Guo et al. reported the BRAF V600E mutation was not associated with cervical lymph node metastasis (LNM), but the BRAF V600E expression had shown significantly associated with cervical LNM [17]. Shu liu et al. reported correlation of BRAF V600E with extrathyroidal tumor invasion in a Chinese PTC population,

Table 3 Coexistence gene mutation of PTC

\begin{tabular}{lll}
\hline $\begin{array}{l}\text { Gene mutation } \\
\text { combination }\end{array}$ & No168 & \\
\cline { 3 - 3 } BRAF+ TERT & No. & \\
BRAF + TERT C228T & 9 & 5.4 \\
BRAF+ TERT C250T & 1 & 0.5 \\
BRAF+ RAS & & \\
BRAF+ HRAS & 3 & 1.8 \\
BRAF+ NRAS & 2 & 1.2 \\
BRAF+ KRAS & 2 & 1.2 \\
BRAF+ TP53 & 3 & 1.8 \\
BRAF+ PIK3CA & 3 & 1.2 \\
BRAF+ AKT1 & 2 & 0.5 \\
BRAF+ PTEN & 1 & 0.5 \\
\hline
\end{tabular}

however, the authors reported no correlation with other clinicopathological features [8]. These different findings might be due to variations in the study cohorts in terms of age distribution, histological variants of tumors, environmental factors and disease staging..

Mutations involving gene fusions in multiple cancers are considered driver events that lead to tumorigenesis, thus providing potential diagnostic markers or targets for precision treatment. We examined gene fusions with $R E T$ and NTRK1 in our study. RET/PTC fusion is the most common type of gene fusions in PTC. RET fusion is considered an early event in PTC tumorigenesis. Radiation exposure has been shown to increase the risk of RET/PTC fusion [18]. Approximately $90 \%$ of reported RET/PTC fusions are RET/PTC1 (CCDC6-RET) and RET/PTC3 (NCOA4-RET) [19], consistent with our findings, which showed a RET fusion percentage of $92.3 \%(12 / 13)$.

NTRK1 fusion with TPM3, TPR or TFG genes are oncogenic in PTC, patients with NTRK1 gene fusion mutations often have a poor prognosis and tend to have younger age [20]. Under the control of the thyroid globulin promoter, TPR-NTRK1 transgenic mice develop thyroid hyperplasia and papillary thyroid cancer [21]. We found two cases of NTRK1 gene fusion mutations in our study, with TPR and $I R F 2 B P 2$ being the fusion partners. Liang et al. reported a case of IRF2BP2-NTRK1 fusion in Chinese patients. It was shown that IRF2BP2-NTRK1 fusion led to a higher expression of NTRK1 tyrosine kinase structural domain [22]. 
Table 4 Relationships between BRAF V600E alone or RET fusion alone or BRAF + TERT or BRAF + RAS mutations and clinicopathological features in PTC patients

\begin{tabular}{|c|c|c|c|c|c|c|c|c|c|c|c|c|}
\hline \multirow[t]{2}{*}{ Characteristics } & \multicolumn{3}{|c|}{ BRAF VGO0E } & \multicolumn{3}{|c|}{ RET Fusion } & \multicolumn{3}{|c|}{$B R A F+T E R T$} & \multicolumn{3}{|c|}{$B R A F+R A S$} \\
\hline & $\begin{array}{l}\text { Positive } \\
(N=119)\end{array}$ & $\begin{array}{l}\text { Negative } \\
(N=49)\end{array}$ & $P$-value & $\begin{array}{l}\text { Positive } \\
(N=13)\end{array}$ & $\begin{array}{l}\text { Negative } \\
(N=155)\end{array}$ & $P$-value & $\begin{array}{l}\text { Positive } \\
(N=10)\end{array}$ & $\begin{array}{l}\text { Negative } \\
(N=158)\end{array}$ & $P$-value & $\begin{array}{l}\text { Positive } \\
(N=7)\end{array}$ & $\begin{array}{l}\text { Negative } \\
(N=161)\end{array}$ & $P$-value \\
\hline \multicolumn{13}{|l|}{ Gender } \\
\hline Female & $92(77.3)$ & 39 (79.6) & 0.746 & $9(71.4)$ & $122(78.6)$ & 0.428 & $7(70.0)$ & $124(78.5)$ & 0.530 & $6(85.7)$ & $125(77.6)$ & 0.614 \\
\hline Male & $27(22.7)$ & $10(20.4)$ & & $4(28.6)$ & $33(21.4)$ & & $3(30.0)$ & $34(21.5)$ & & $1(14.3)$ & $36(22.4)$ & \\
\hline \multicolumn{13}{|l|}{ Age } \\
\hline$<45$ & $73(61.3)$ & $34(69.4)$ & 0.324 & $11(85.7)$ & $96(61.7)$ & 0.102 & $2(20.0)$ & $105(66.5)$ & $0.003^{*}$ & $5(71.4)$ & $102(63.4)$ & 0.679 \\
\hline$\geq 45$ & $46(38.7)$ & 15 (30.6) & & $2(14.3)$ & $59(38.3)$ & & $8(80.0)$ & $53(33.5)$ & & $2(28.6)$ & $58(36.6)$ & \\
\hline \multicolumn{13}{|l|}{ Subtypes } \\
\hline conventional PTC & $116(97.5)$ & $40(81.6)$ & $<0.001^{*}$ & $11(85.7)$ & $145(93.5)$ & 0.230 & $10(100)$ & $146(92.4)$ & 0.366 & $7(100)$ & $149(92.5)$ & 0.454 \\
\hline follicular variant PTC & $3(2.5)$ & $9(18.4)$ & & $2(14.3)$ & $10(6.5)$ & & $0(0)$ & $12(7.6)$ & & $0(0)$ & $12(7.5)$ & \\
\hline \multicolumn{13}{|l|}{ Lymph node metastasis } \\
\hline Yes & 107 (89.9) & $42(85.7)$ & 0.434 & $12(92.9)$ & $137(88.3)$ & 0.668 & $9(90.0)$ & 140 (88.6) & 0.893 & $6(85.7)$ & $143(88.8)$ & 0.800 \\
\hline No & $12(10.1)$ & $7(24.3)$ & & $1(7.1)$ & $18(11.7)$ & & $1(10.0)$ & $18(11.4)$ & & $1(14.3)$ & $18(11.2)$ & \\
\hline \multicolumn{13}{|l|}{ AJCC disease stage } \\
\hline $\mid+\|$ & 75 (63.0) & $34(69.4)$ & 0.432 & $11(85.7)$ & $98(63.0)$ & 0.121 & $2(20.0)$ & $107(67.7)$ & $0.002^{*}$ & $5(71.4)$ & $104(64.6)$ & 0.711 \\
\hline$I I I+I V$ & $44(37.0)$ & 15 (30.6) & & $2(14.3)$ & $57(37.0)$ & & $8(80.0)$ & $51(32.3)$ & & $2(28.6)$ & $57(35.4)$ & \\
\hline \multicolumn{13}{|l|}{ Lesion number } \\
\hline Single lesion & $54(55.5)$ & $24(75.5)$ & 0.671 & $10(76.9)$ & $93(60.0)$ & 0.655 & $2(20.0)$ & $76(63.9)$ & 0.084 & $0(28.6)$ & $78(62.7)$ & $0.015^{*}$ \\
\hline Multiple lesions & $65(44.5)$ & $25(24.5)$ & & $3(23.1)$ & $62(40.0)$ & & $8(80.0)$ & $82(36.1)$ & & $7(71.4)$ & $83(37.3)$ & \\
\hline
\end{tabular}

Values are presented as number (\%). ${ }^{*} p<0.05$. BRAF+RAS means BRAF+NRAS and BRAF+HRAS and BRAF+KRAS dual mutations together

In our current study, we did not see NTRK1 fusion correlated with patient clinicopathologic features.

TERT promoter mutations are relatively common in PTC, affecting approximately $10 \%$ of all PTC, with C228T being the most dominant mutation and C250T mutations making up a smaller percentage [5]. TERT promoter mutations have been associated with aggressive tumor behaviors and worse prognosis in thyroid cancer [23]. In a large study of 1892 PTC patients, it was found that BRAF V600E and TERT promoter mutations coexist in $7.7 \%$ of all primary PTC [24]. While each type of mutation alone had a modest adverse effect, the double mutations were associated with much worse clinicopathologic outcomes, including extrathyroidal invasion, lymph node metastasis, distant metastasis, and disease recurrence [9]. In our study, we identified 11 cases with TERT promoter mutations, with 10 cases of C228T mutation, and 1 case of C250T mutation. Among 11 cases with TERT promoter mutations, 10 cases also had BRAF V600E mutation, and 1 case had NRAS mutation. In our study, BRAF V600E and TERT promoter mutations coexist in $6 \%$ of all PTC, in the same range as the previous report [24]. We found that BRAF V600E and TERT promoter mutation duet was associated with older patient age $(>45, p=0.003)$ and higher disease stage of III or IV $(p=0.002)$.
In our cohort of high-recurrence risk PTC patients, we found multiple cases of dual mutations of BRAF V600E together with another mutation, including TERT, RAS, TP53, PIK3CA, AKT1, and PTEN. While mutation duet of BRAF V600E and TERT were most common, we unexpectedly identified 7 cases with $B R A F$ V600E and $R A S$ dual mutations. $R A S$ mutations have been seen in several thyroid cancer types, including follicular thyroid cancer, poorly differentiated thyroid cancer, undifferentiated thyroid cancer and PTC [25]. Xing et al. reported that $R A S$ mutation alone does not indicate malignancy in thyroid tumors [26]. However, thyroid cancer with dual mutations of RAS with BRAF V600E or TERT was associated with worse clinicopathologic outcomes [11, 27]. In our current study, dual mutations of $R A S$ and $B R A F$ V600E were only seen in multifocal PTC $(p=0.015)$.

In conclusion, in our study of high-recurrent risk PTC, we saw a high prevalence of BRAF V600E mutation (70.8\%). BRAF V600E and TERT dual mutations were associated with older patient age $(>45)$ and higher disease stage. RAS and BRAF V600E dual mutations were also seen in this patient cohort and were associated with multifocal disease. In general, RAS and BRAF V600E mutations tend to be mutually exclusive, however, there have been reports of their coexistence in PTC $[11,28]$. Whether their 
coexistence affects clinicopathologic outcomes of PTC remains to be studied further.

\section{Supplementary information}

Supplementary information accompanies this paper at https://doi.org/10. 1186/s13000-020-00962-8.

Additional file 1.

Additional file 2.

\section{Abbreviations}

PTC: Papillary Thyroid Carcinoma; FFPE: Formalin-fixed paraffin-embedded; MAPK: Mitogen-associated protein kinase; NGS: Next-generation sequencing; PCR: Polymerase Chain Reaction; QC: Quality Control; TNM: Tumor Node Metastasis; AJCC: American Joint Committee on Cancer; LNM: Lymph node metastasis; BRAF: B-type Raf kinase; TERT: Telomerase reverse transcriptase; TP53: Tumor protein p53; RET: Rearranged during transfection; PIK3CA: PI3K subunit p110alpha; PTEN: Phosphataseandtensinhomolog; NTRK1: Neurotrophic tyrosine kinase, receptor, type 1

\section{Acknowledgments}

Not applicable.

\section{Authors' contributions}

FS and WYZ designed the study; ML and HTJ wrote the manuscript; ML and QQQ collected samples and clinical information; PW and CC performed the experiments and acquired data; $\mathrm{YQH}$ and KW analyzed the data and drew the picture; all authors revised and approved the manuscript.

\section{Funding}

Not applicable.

\section{Availability of data and materials}

The datasets used and/or analyzed during the current study are available from the corresponding author upon reasonable request.

\section{Ethics approval and consent to participate}

The ethics committee of Hunan Cancer Hospital passed ethical approval of this study.

\section{Consent for publication}

Written informed consent for publication was obtained from each participant.

\section{Competing interests}

The authors declare that they have no conflicts of interest.

\section{Author details}

${ }^{1}$ Department of Nuclear Medicine, The Affiliated Cancer Hospital of Xiangya School of Medicine, Central South University, and Hunan Cancer Hospital, Changsha 410013, China. ${ }^{2}$ Shenzhen Cheerland Biotechnology Co., Ltd, Shenzhen 518055, China. ${ }^{3}$ School of Medicine, Southern University of Science and Technology, Shenzhen 518055, China.

Received: 13 November 2019 Accepted: 28 April 2020

Published online: 11 May 2020

\section{References}

1. Carling T, Udelsman R. Thyroid Cancer. Annu Rev Med. 2014;65(1):125-37. https://doi.org/10.1146/annurev-med-061512-105739.

2. Mao $Y$, Xing $M$. Recent incidences and differential trends of thyroid cancer in the USA. Endocr Relat Cancer. 2016;23(4):313-22. https://doi.org/10.1530/ ERC-15-0445.

3. Wang $Y$, Wang W. Increasing incidence of thyroid cancer in Shanghai, China, 1983-2007. Asia Pac J Public Health. 2015;27(2):NP223-9. https://doi. org/10.1177/1010539512436874.

4. Huang Y, Qu S, Zhu G, Wang F, Liu R, Shen X, et al. BRAF V600E mutationassisted risk stratification of solitary Intrathyroidal papillary thyroid Cancer for precision treatment. J Natl Cancer Inst. 2018;110(4):362-70. https://doi. org/10.1093/jnci/djx227.

5. Cancer Genome Atlas Research N. Integrated genomic characterization of papillary thyroid carcinoma. Cell. 2014;159(3):676-90. https://doi.org/10. 1016/j.cell.2014.09.050.

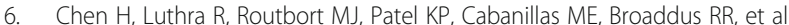
Molecular profile of advanced thyroid carcinomas by next-generation sequencing: characterizing tumors beyond diagnosis for targeted therapy. Mol Cancer Ther. 2018;17(7):1575-84. https://doi.org/10.1158/1535-7163. MCT-17-0871.

7. Xing M. BRAF mutation in papillary thyroid cancer: pathogenic role, molecular bases, and clinical implications. Endocr Rev. 2007;28(7):742-62. https://doi.org/10.1210/er.2007-0007.

8. Liu S, Zhang B, Zhao Y, Chen P, Ji M, Hou P, et al. Association of BRAF V600E mutation with clinicopathological features of papillary thyroid carcinoma a study on a Chinese population. Int J Clin Exp Pathol. 2014; 7(10):6922-8.

9. Shi X, Liu R, Qu S, Zhu G, Bishop J, Liu X, et al. Association of TERT promoter mutation 1,295,228 C>T with BRAF V600E mutation, older patient age, and distant metastasis in anaplastic thyroid cancer. J Clin Endocrinol Metab. 2015:100(4):E632-7. https://doi.org/10.1210/jc.2014-3606.

10. Xing M. Molecular pathogenesis and mechanisms of thyroid cancer. Nat Rev Cancer. 2013;13(3):184-99. https://doi.org/10.1038/nrc3431.

11. Huang M, Yan C, Xiao J, Wang T, Ling R. Relevance and clinicopathologic relationship of BRAF V600E, TERT and NRAS mutations for papillary thyroid carcinoma patients in Northwest China. Diagn Pathol. 2019;14(1):74. https:// doi.org/10.1186/s13000-019-0849-6.

12. Medas F, Canu GL, Boi F, Lai ML, Erdas E, Calo PG. Predictive Factors of Recurrence in Patients with Differentiated Thyroid Carcinoma: A Retrospective Analysis on 579 Patients. Cancers (Basel). 2019;11(9). https:// doi.org/10.3390/cancers11091230

13. Basolo F, Torregrossa L, Giannini R, Miccoli M, Lupi C, Sensi E, et al. Correlation between the BRAF V600E mutation and tumor invasiveness in papillary thyroid carcinomas smaller than 20 millimeters: analysis of 1060 cases. J Clin Endocrinol Metab. 2010;95(9):4197-205. https://doi.org/10.1210/ jc.2010-0337.

14. Li C, Lee KC, Schneider EB, Zeiger MA. BRAF V600E mutation and its association with clinicopathological features of papillary thyroid cancer: a meta-analysis. J Clin Endocrinol Metab. 2012;97(12):4559-70. https://doi.org/ 10.1210/jc.2012-2104.

15. Wada N, Masudo K, Nakayama H, Suganuma N, Matsuzu K, Hirakawa S, et al. Clinical outcomes in older or younger patients with papillary thyroid carcinoma: impact of lymphadenopathy and patient age. Eur J Surg Oncol. 2008;34(2):202-7. https://doi.org/10.1016/j.ejso.2007.10.001.

16. Zhang B, Xu CW, Wu YF, Man QH, Song YY, Wang JJ, et al. Diagnostic significance of the BRAF V600E mutation in conventional papillary thyroid carcinomas. Int J Clin Exp Med. 2016:9(5):8296-303.

17. Guo L, Ma YQ, Yao Y, Wu M, Deng ZH, Zhu FW, et al. Role of ultrasonographic features and quantified BRAFV600E mutation in lymph node metastasis in Chinese patients with papillary thyroid carcinoma. Sci Rep. 2019:9(1):75. https://doi.org/10.1038/s41598-018-36171-z.

18. Prescott JD, Zeiger MA. The RET oncogene in papillary thyroid carcinoma. Cancer. 2015:121(13):2137-46. https://doi.org/10.1002/cncr.29044.

19. Carlomagno F. Thyroid Cancer: role of RET and beyond. Eur Thyroid J. 2012; 1(1):15-23. https://doi.org/10.1159/000336975.

20. Greco A, Miranda C, Pierotti MA. Rearrangements of NTRK1 gene in papillary thyroid carcinoma. Mol Cell Endocrinol. 2010;321(1):44-9. https://doi.org/10. 1016/j.mce.2009.10.009.

21. Russell JP, Powel DJ, Cunnane M, Greco A, Portella G, Santoro M, et al. The TRK-T1 fusion protein induces neoplastic transformation of thyroid epithelium. Oncogene. 2000;19:5729-35. https://doi.org/10.1038/sj.onc. 1203922.

22. Liang J, Cai W, Feng D, Teng H, Mao F, Jiang Y, et al. Genetic landscape of papillary thyroid carcinoma in the Chinese population. J Pathol. 2018;244(2): 215-26. https://doi.org/10.1002/path.5005

23. Jin $L$, Chen $E$, Dong $S$, Cai $Y$, Zhang $X$, Zhou $Y$, et al. BRAF and TERT promoter mutations in the aggressiveness of papillary thyroid carcinoma a study of 653 patients. Oncotarget. 2016;7(14):18346-55. https://doi.org/10. 18632/oncotarget.7811.

24. Liu R, Bishop J, Zhu G, Zhang T, Ladenson PW, Xing M. Mortality risk stratification by combining BRAF V600E and TERT promoter mutations in 
papillary thyroid Cancer: genetic duet of BRAF and TERT promoter mutations in thyroid Cancer mortality. JAMA Oncol. 2017:3(2):202-8. https:// doi.org/10.1001/jamaoncol.2016.3288.

25. Howell GM, Hodak SP, Yip L. RAS mutations in thyroid cancer. Oncologist. 2013;18:926-32.

26. Xing M. Clinical utility of RAS mutations in thyroid cancer: a blurred picture now emerging clearer. BMC Med. 2016;14:12. https://doi.org/10.1186/ s12916-016-0559-9.

27. Sohn SY, Park WY, Shin HT, Bae JS, Ki CS, Oh YL, et al. Highly concordant key genetic alterations in primary tumors and matched distant metastases in differentiated thyroid Cancer. Thyroid. 2016;26(5):672-82. https://doi.org/ 10.1089/thy.2015.0527.

28. Zou M, Baitei EY, Alzahrani AS, BinHumaid FS, Alkhafaji D, Al-Rijjal RA, et al. Concomitant RAS, RET/PTC, or BRAF mutations in advanced stage of papillary thyroid carcinoma. Thyroid. 2014;24(8):1256-66. https://doi.org/10. 1089/thy.2013.0610.

\section{Publisher's Note}

Springer Nature remains neutral with regard to jurisdictional claims in published maps and institutional affiliations.

Ready to submit your research? Choose BMC and benefit from:

- fast, convenient online submission

- thorough peer review by experienced researchers in your field

- rapid publication on acceptance

- support for research data, including large and complex data types

- gold Open Access which fosters wider collaboration and increased citations

- maximum visibility for your research: over $100 \mathrm{M}$ website views per year

At BMC, research is always in progress.

Learn more biomedcentral.com/submissions 